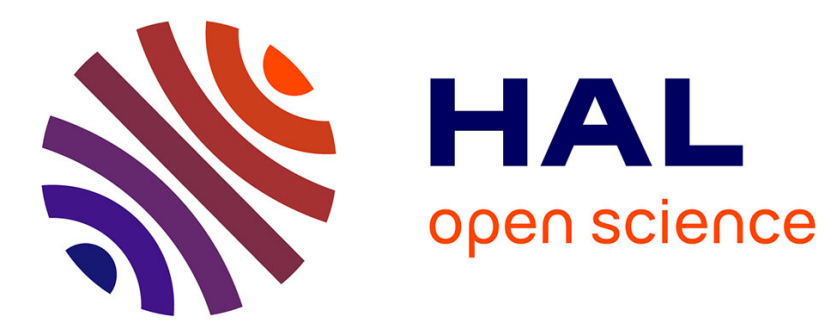

\title{
Application of global sensitivity analysis to a tire model with correlated inputs
}

Floriane Anstett-Collin, Thierry A. Mara, Michel Basset

\section{To cite this version:}

Floriane Anstett-Collin, Thierry A. Mara, Michel Basset. Application of global sensitivity analysis to a tire model with correlated inputs. Simulation Modelling Practice and Theory, 2014, 44, pp.54-62. 10.1016/j.simpat.2014.03.003 . hal-00958652

\section{HAL Id: hal-00958652 https://hal.science/hal-00958652}

Submitted on 13 Mar 2014

HAL is a multi-disciplinary open access archive for the deposit and dissemination of scientific research documents, whether they are published or not. The documents may come from teaching and research institutions in France or abroad, or from public or private research centers.
L'archive ouverte pluridisciplinaire HAL, est destinée au dépôt et à la diffusion de documents scientifiques de niveau recherche, publiés ou non, émanant des établissements d'enseignement et de recherche français ou étrangers, des laboratoires publics ou privés. 


\title{
Application of global sensitivity analysis to a tire model with correlated inputs
}

\author{
Floriane Anstett-Collin ${ }^{\mathrm{a}, *}$, Thierry Mara ${ }^{\mathrm{b}}$, Michel Basset $^{\mathrm{c}}$ \\ ${ }^{a}$ Université de Lorraine - 2 Rue Jean Lamour - 54519 Vandoeure Lès Nancy Cedex, \\ France \\ ${ }^{b}$ Université de La Réunion - 15 Avenue René Cassin - 97715 Saint-Denis Messag Cedex \\ 9, La Réunion \\ ${ }^{c}$ Université de Haute Alsace - 12 rue des frères Lumière - 68093 Mulhouse Cedex, France
}

\section{Abstract}

When a vehicle equipped with tire is manoeuvred on the ground, the tires are submitted to a number of forces - longitudinal force when driving or braking torque is applied to the wheel and/or lateral force when the wheel is steered to turn at a corner. Pacejka model describes these forces that represent the reaction of the road onto the tire. This nonlinear model depends on correlated parameters such as the friction coefficient, the vertical load, the cornering stiffness, ... which have to be identified from some measurements. The sensitivity of Pacejka model to these correlated parameters are studied using an approach based on polynomial chaos. It consists in decorrelating the parameters using the Nataf transformation and then, in expanding the model output onto polynomial chaos. The sensitivity indices are then obtained straightforwardly from the algebraic expression of the coefficients of the polynomial expansion.

Keywords: Global sensitivity analysis, correlated parameters, polynomial

\footnotetext{
*Corresponding author: floriane.collin@univ-lorraine.fr
} 
chaos expansion, tire model

\section{Introduction}

In the automotive and aeronautical fields, modelling the tire/road interface is fundamental. Indeed, the tire model is one of the major elements to integrate into a ground vehicle or aircraft model, as the tires are the only contact surface with the road. When a vehicle equipped with tires is manoeuvred on the ground, they are subject to a number of forces - longitudinal force when driving or braking torque is applied to the wheel and lateral force when the wheel is steered to turn at a corner. The tire models available in the literature describe the efforts and moments corresponding to the road reaction of the tire (see for instance $[1,2,3,4]$ ).

One of the most famous model was proposed by Pacejka ([1]) and is often used ([5]) nowadays by industrials (Michelin, SAE, Adams tire software, ...). This model is nonlinear, complex and depends on a certain number of parameters (friction coefficient, vertical load, side slip angle, ...) that can be obtained from experimentation. Unfortunately, experimental data are often sparse or incomplete, especially in the aircraft domain and their measurements are very expensive. On the one hand, some parameters only have a negligible influence on the model response and therefore, do not need to be determined accurately. On the other hand, some others are relevant for the model response and thus influence its uncertainty significantly. These parameters may require additional measurement effort in order to be estimated with relatively high accuracy. In order to prepare and plan future experiments, it is necessary to perform a sensitivity analysis of the Pacejka 
model.

Several studies have focused on the global sensitivity analysis of nonlinear models with independent inputs (for instance, $[6,7,8,9,10,11,12]$ ). They rely on variance-based sensitivity indices also known as Sobol' indices ([7]). They measure the contribution of input parameters to the model response variance. Such a contribution can be due to a parameter alone or to a group of parameters. Sobol' indices are unique and easily interpretable when the parameters are independent. But, it is more challenging when they are dependent. Indeed, if a function (i.e. model response) structurally depends on parameter $p_{1}$ and not on $p_{2}$, and if $p_{1}$ and $p_{2}$ are correlated, classical Sobol' indices will lead to the conclusion that both are relevant inputs. Even though actually $p_{2}$ is influential only because of its correlation with $p_{1}$.

It may be of high interest to distinguish whether an input is relevant regarding its correlations with the other ones [13]. This information may be of great help for the experimenter in order to guide future experiments. Mara and Tarantola ([14]) have derived a set of variance-based sensitivity indices to cope with parameters dependency. They also proposed a computational method for their estimation. The proposed approach relies on the use of polynomial chaos expansion ([15]) in conjunction with a GramSchmidt based decorrelation procedure. They also indicate that Nataf ([16]) or Rosenblatt ([17]) transformations can be used.

This study is part of a french national project involving several industrial partners (Airbus, Messier-Dowty, Turbomeca, etc) and research centers, in particular the Modélisation Intelligence Processus Systèmes (MIPS) laboratory. The aim of the project is to take advantage of performances of the 
modern simulation tools by providing new models and developing tools which allow the simulation of systems and physical phenomenon in the aeronautical fields. More precisely, the laboratory works to develop models for simulating the tyre-road interaction characteristics with respect to the aircraft run types. In this framework, the aim of the paper is to analyze the well-known Pacejka tire model, which is a basic function of the Magic Formula ([1]). Pacejka model is widely used in the automotive and aeronautical fields. The model depends on correlated parameters (friction coefficient, cornering stiffness, vertical load, ...) which must be identified from measurement data. The most relevant parameters on the lateral force are highlighted, using the approach proposed in [14].

The paper is organised as follows. Section 2 presents the Pacejka tire model. Section 3 recalls the expression of the Sobol' sensitivity indices for model with independent parameters and their estimation based on polynomial chaos. Section 4 presents the approach used to study the sensitivity for models with correlated parameters. Then, the sensitivity analysis of the Pacejka tire model is performed.

\section{Pacejka tire model}

Tires in motion on the ground are subject to a number of forces. For example, a longitudinal force is developed when driving or braking torque is applied to the wheel. A lateral force appears when the wheel is at an angle or when it is steered to turn at a corner. The model considered in this study accounts for the longitudinal force (driving or braking force) and the lateral

force. In the present study, we will exclusively focus on the model expression 
for the lateral force developed in the case of a cornering manoeuvre in steadystate condition, as represented in figure 1.
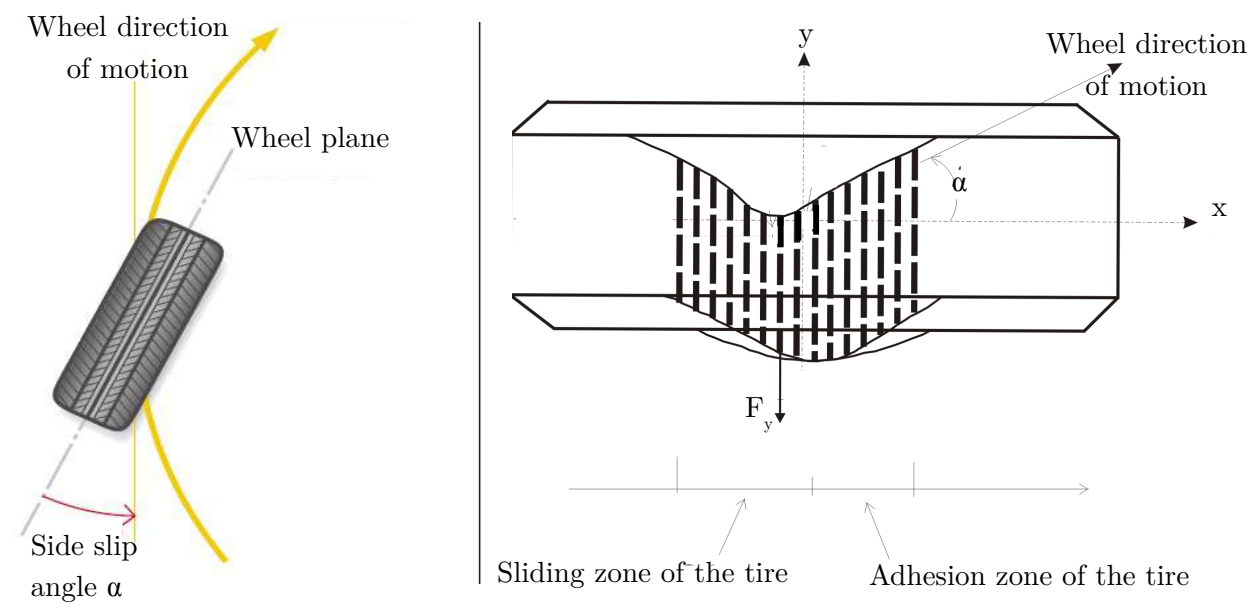

Figure 1: Pure cornering - General view of the tire deformation in the contact patch

In pure cornering condition, an interpolation function, called Magic Formula ([1]), is proposed for the lateral force. The Pacejka model presented here is a basic function of the Magic Formula. In this case, the lateral force is given by:

$$
F_{y}=D \sin \left[C \arctan \left(B\left(\alpha+S_{h}\right)-E\left(B\left(\alpha+S_{h}\right)-\arctan \left(B\left(\alpha+S_{h}\right)\right)\right)\right)\right]+S_{V}
$$

The model output of interest $F_{y}$ is the lateral force, road reaction in the lateral direction. The parameter $\alpha$ is the side slip angle, that is the angle between the wheel plane and the wheel direction of motion (see figure 1). 
The parameter $D$ represents the maximum value that $F_{y}$ can reach and $C, E$, $S_{h}$ and $S_{v}$ are empirical fitting parameters. Figure 2 depicts the relationship between the lateral force $F_{y}$ and the side slip angle $\alpha$. In figure 2, the product $B \times C \times D$, corresponding to the cornering stiffness, is the slope at the origin.

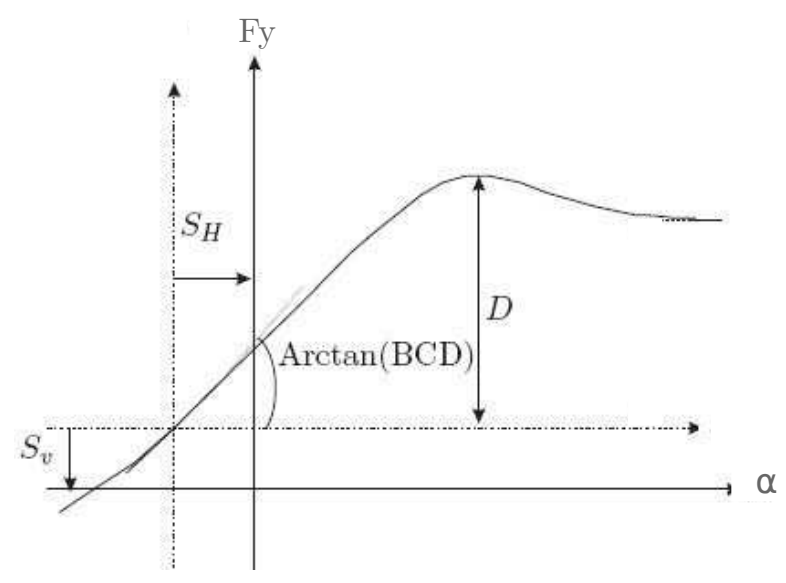

Figure 2: Lateral force vs. side slip angle

The parameters $E, S_{h}$ and $S_{v}$ are known and set as follows, $E=0.2, S_{h}=$ 0.003 and $S_{v}=3000$. These are common values in the aeronautical field. The parameters $B, C, D$ and $\alpha$ are uncertain. The aim of this study is to determine the contribution of $B, C, D$ and $\alpha$ to the variation of $F_{y}$. The set of parameters $(B, C, D)$ is dependent since $B$ and $D$ are expressed as:

$$
B=\frac{K}{D C}, \quad D=-3.4 \times 10^{-7} F_{z}^{2}+0.74 F_{z} .
$$


where $K$ is the cornering stiffness and $F_{z}$ the vertical load. The ratio $D / F_{z}$ represents the lateral friction coefficient $\mu$ that characterizes the road state. The uncertain parameters $F_{z}, \alpha, C$ and $K$ are independent. The ultimate aim of this study is to perform the sensitivity analysis of $F_{y}$ to the parameter set $(B, C, D, \alpha)$ because the model response directly depends on these four macro-parameters. But, let first analyze the contribution of the independent parameters $\left(\alpha, F_{z}, K, C\right)$ on $F_{y}$.

Data collected from the aeronautical field experimentation during the MIPS project framework and specifications from manufacturer leads to the distributions of the parameters $C, \alpha, K, F_{Z}$, given in Table 1 . All parameters follow a normal distribution with mean and standard deviation given in Table 1.

\begin{tabular}{|c|c|}
\hline Parameters & Normal distributions: $\mathcal{N}($ mean; standard deviation $)$ \\
\hline$C$ & $\mathcal{N}(1.5 ; 0.1)$ \\
\hline$\alpha$ & $\mathcal{N}(0.1739 ; 0.0360)$ \\
\hline$K$ & $\mathcal{N}\left(6.7208 \times 10^{5} ; 9.7010 \times 10^{4}\right)$ \\
\hline$F_{z}$ & $\mathcal{N}\left(9.0034 \times 10^{4} ; 5.5803 \times 10^{3}\right)$ \\
\hline
\end{tabular}

Table 1: Distribution of the independent parameters

The next section recalls the expression of the Sobol' indices for models with independent parameters. 


\section{Sensitivity analysis for models with independent inputs}

\subsection{Sobol' indices}

Let $y=f(\mathbf{p})$ be a square integrable function of a set of $n$ independent random inputs $\mathbf{p}=\left(p_{1}, \ldots, p_{n}\right) \in \mathbb{R}^{n}$. Sobol' proved that $f$ can be expanded as a sum of orthogonal functions of increasing dimensionality:

$$
f(\mathbf{p})=f_{0}+\sum_{i=1}^{n} f_{i}\left(p_{i}\right)+\sum_{j>i}^{n} f_{i j}\left(p_{i}, p_{j}\right)+\ldots+f_{1 \cdots n}\left(p_{1}, \cdots, p_{n}\right)
$$

and that this decomposition is unique ([7]).

Thanks to the orthogonality of the functions in equation (3), it is straightforward to decompose the variance of $y$, denoted $V_{y}$ :

$$
V_{y}=\sum_{i=1}^{n} V_{i}+\sum_{j>i}^{n} V_{i j}+\ldots+V_{1 \cdots n}
$$

with:

$$
V_{i_{1} \ldots i_{s}}=\operatorname{Var}\left(f_{i_{1} \ldots i_{s}}\right)
$$

where $V a r$ is the variance operator.

As explained in [7], the Sobol' sensitivity indices are obtained by renormalising (5) with the total variance $V_{y}$ :

$$
S_{i_{1} \ldots i_{s}}=\frac{V_{i_{1} \ldots i_{s}}}{V_{y}}, \quad 1 \leq i_{1} \leq \ldots \leq i_{s} \leq n .
$$

$S_{i_{1} \ldots i_{s}}$ represents the amount of $V_{y}$ due to the interaction between $\left(p_{i_{1}}, \ldots, p_{i_{s}}\right)$. In particular, the first-order sensitivity index, denoted $S_{i}$, that represents the individual contribution of the parameter $p_{i}$ to the variance of $y$, writes:

$$
S_{i}=\frac{V_{i}}{V_{y}}
$$


Finally, we define the total sensitivity index of parameter $p_{i}$, denoted $S T_{i}$, that includes its individual as well as its collective contributions to $V_{y}$ as follows:

$$
S T_{i}=\sum_{i \in \mathbf{u}_{i} \subseteq\{1, \ldots, n\}} S_{\mathbf{u}_{i}}
$$

As a result, $S_{i} \leq S T_{i} \in[0,1]$. A parameter is irrelevant if $S T_{i}=0$ and does not interact with the other parameters if $S T_{i}=S_{i}$. The previous sensitivity indices can be calculated using Monte Carlo simulations ([7]) or Fourier analysis ([8]). Using Monte Carlo simulations with a sample size of $N$ for a model with $n$ parameters leads to $N \times 2^{n}$ evaluations of the model function to compute all the indices. This can be very costly to manage. An alternative consists in casting the orthogonal functions in expansion (3) onto orthogonal polynomials known as polynomial chaos (PC). Indeed, it was shown in $[18,19]$ that the sensitivity indices could be evaluated as analytical expressions of the PC coefficients. Hence, the cost of computing the sensitivity indices is the one of evaluating the PC coefficients and requires only $N$ model evaluations.

\subsection{Polynomial chaos expansion (PCE)}

In [15], it was shown that the homogeneous chaos expansion could be used to approximate any function in the Hilbert space of square-integrable functions. Therefore, the model output $y$ can be decomposed as follows:

$$
y=\sum_{j=0}^{+\infty} \alpha_{j} \Phi_{j}(\mathbf{p})
$$

where the multivariate polynomial $\Phi_{j}$ of degree $j$ is given by the tensor product of the corresponding one-dimensional Hermite polynomials $\phi_{a_{k}^{j}}$ :

$$
\Phi_{j}(\xi)=\prod_{k=1}^{n} \phi_{a_{k}^{j}}\left(p_{k}\right)
$$


with $a_{k}^{j} \in \mathbb{N}$ the degree of $\phi_{a_{k}^{j}}$, such that $\left|a^{j}\right|=\sum_{k=1}^{n} a_{k}^{j}$. The theorem of Cameron and Martin ([20]), ensures that an expansion as (9) converges in the $\mathcal{L}_{2}$-sense. The convergence rate is optimal when the parameters are normally distributed. For other parameters distribution, the authors in [21] introduced the generalized polynomial chaos to ensure an optimal convergence rate (Legendre polynomials for uniform distribution, Jacobi polynomials for Beta distribution, ....).

In practice, the PC expansion (9) is truncated up to a finite degree $d$ and the number of coefficients in the expansion equals:

$$
M+1=\frac{(n+d) !}{n ! d !}
$$

The optimal degree $d$ could be selected by incrementing its value until a target accuracy, for instance the determination coefficient $R^{2}$, is reached. However, this can lead to a large number $N$ of model evaluations to compute the PC coefficients when increasing $d$ because $N$ has to be greater than $M$. To overcome this problem, an adaptive scheme has been proposed in [22] to build up a sparse PC expansion.

The deterministic PC coefficients (i.e. the $\alpha_{j}$ 's) are the unknowns and several non-intrusive approaches are proposed in the literature to compute them. They can be classified as regression techniques (e.g. [18, 23, 24]) or projection techniques (for instance [19, 25]). In the case of the regression method, it is generally advisable to use an over-sampling to determine the sample size $N$, resulting in a least squares solution for the over-determined system. It is proposed to use $N=2(M+1)$ in [26] and $N=(n-1)(M+1)$, in [18], stating 
that taking more points does not improve the accuracy of the results. In light of the dependence of $M$ on the PC order and the number $n$ of parameters, the $\mathrm{PC}$ representation will be computationally efficient when small values of $N$ and $d$ are sufficient for an accurate representation of the random variables.

The computation of Sobol' indices is straightforward once computed the PC coefficients. Indeed, let define $\mathcal{I}_{k_{1}, \ldots, k_{s}}$ the set of multi-indices $j$ such that:

$$
\mathcal{I}_{k_{1}, \ldots, k_{s}}=\left\{0 \leq a_{k}^{j} \leq d, a_{k}^{j}=0 \forall k \in\{1, \ldots, n\} \backslash\left\{k_{1}, \ldots, k_{s}\right\}\right\}
$$

The first-order sensitivity index $S_{i}$ of parameter $p_{i}$ is estimated as follows:

$$
\hat{S}_{i}=\frac{\sum_{j \in \mathcal{I}_{i}} \alpha_{j}^{2} E\left(\Phi_{j}^{2}\left(p_{i}\right)\right)}{\sum_{j=1}^{M} \alpha_{j}^{2} E\left(\Phi_{j}^{2}(\mathbf{p})\right)}
$$

where $E$ is the expectation operator and the set $\mathcal{I}_{i}$ corresponds to the polynomials depending on $p_{i}$ only, with the exception of all the others. Higher order sensitivity indices can be obtained in the same manner:

$$
\hat{S}_{i_{1}, \ldots, i_{s}}=\frac{\sum_{j \in \mathcal{I}_{i_{1}, \ldots, i_{s}}} \alpha_{j}^{2} E\left(\Phi_{j}^{2}\left(p_{i_{1}}, \ldots, p_{i_{s}}\right)\right)}{\sum_{j=1}^{M} \alpha_{j}^{2} E\left(\Phi_{j}^{2}(\mathbf{p})\right)}
$$

The total sensitivity index is given by:

$$
\hat{S T_{i}}=\frac{\sum_{j \in \mathcal{I}_{i^{+}}} \alpha_{j}^{2} E\left(\Phi_{j}^{2}\left(p_{i}\right)\right)}{\sum_{j=1}^{M} \alpha_{j}^{2} E\left(\Phi_{j}^{2}(\mathbf{p})\right)}
$$


with $\mathcal{I}_{i^{+}}$the set of multi-indices $j$ defined by:

$$
\mathcal{I}_{i^{+}}=\left\{0 \leq a^{j} \leq p, \quad a_{k}^{j} \neq 0, \quad \forall k=i\right\}
$$

The set $\mathcal{I}_{i^{+}}$corresponds to polynomials $\Phi_{i}$ depending on parameter $p_{i}$ and possibly on the other parameters.

It can be worth noting that sensitivity indices can be computed from the PC coefficients with almost no additional cost. Indeed, only elementary mathematical operations are needed to compute the indices from the PC coefficients.

\subsection{Sensitivity of Pacejka model to $\left(\alpha, F_{z}, K, C\right)$}

\subsubsection{Numerical details}

Consider the set of independent parameters $\mathbf{p}=\left(\alpha, F_{z}, K, C\right)$. Samples of parameters are generated using the well-known Latin Hypercube Sampling (LHS)[27, 28]. Alternative sampling methods exist in the literature (maximin LHS, low discrepancy sequences $[29,30], \ldots)$ but LHS is particularly well adapted to create correlated samples as shown in [31]. Besides, it is easy to implement.

As these parameters are normally distributed, Hermite polynomials are employed. The number of parameters is $n=4$ and a third degree PC expansion is investigated $(d=3)$. Consequently, the number of terms in the decomposition (number of unkowns) is $M+1=35$, according to Eq.(11). The 35 coefficients $\alpha_{j}$ are determined by least-squares regression after evaluating the model with a LHS of size $N=4096$. With a third degree PC, the determination coefficient is of 0.9994 . Moreover, the relative error between the 
model response computed using Pacejka model directly vs using PC decomposition does not exceed $4 \%$, showing good accuracy of the results. To avoid overfitting, the variances of the Pacejka model response and of the PCE are compared (the ratio is of 0.9786 ). This shows that a third degree $\mathrm{PC}$ is sufficient here.

The sensitivity indices are computed using equations (13)-(15). The results are summed up in Table 2.

\subsubsection{Results and discussion}

\begin{tabular}{|c|c|c|c|}
\hline$\alpha$ & $F_{z}$ & $K$ & $C$ \\
\hline$\hat{S}_{\alpha}$ & $\hat{S}_{F_{z}}$ & $\hat{S}_{K}$ & $\hat{S}_{C}$ \\
0.45 & 0.31 & 0.17 & 0.02 \\
\hline$\hat{S T_{\alpha}}$ & $\hat{S T}_{F_{z}}$ & $\hat{S T_{K}}$ & $\hat{S T_{C}}$ \\
0.49 & 0.33 & 0.21 & 0.02 \\
\hline
\end{tabular}

Table 2: First-order and total sensitivity indices

The side slip angle is the most influential parameter on the lateral force of Pacejka model, followed by $F_{z}$ and $K$, while $C$ is not relevant. Consequently, the fitting parameter $C$ can be set at a nominal value of its interval of variation, with no consequence on the variance of $F_{y}$. As a result, the parameter $F_{z}$ determines the maximum value that the lateral force $F_{y}$ can reach for a given side slip angle $\alpha$ while $K$ determines the lateral deflection of the tyre. This can explain the relative importance of these parameters. These indices are consistent with the results expected in the tire domain. Note that, the sum of the first-order indices is about 0.95 which means that 
the contribution due to the interactions is low or put in other words, the model is almost additive.

However, the lateral force depends directly on the macro-parameters $(B, C, D)$. Hence, the analysis of the contribution of the dependent parameter set $(B, C, D, \alpha)$ is of high interest for the designer. The next section is focused on sensitivity analysis of models with correlated parameters.

\section{Sensitivity analysis for models with correlated parameters}

Let us consider now a model $f$ with non normally distributed and correlated parameters. We assume that the parameters are defined by a Gaussian copula characterized by a correlation matrix $\rho$ assumed known. We further assume that the parameters margins $h_{i}$ (margin of $p_{i}$ ) and the associated cumulative density functions $(\mathrm{CDF}) H_{i}$ are also given.

In [14], it is shown that several PC expansions (and consequently ANOVA decompositions) of the model response can be performed by firstly decorrelated the inputs with the Nataf transformation. These PCEs lead to the definition of variance-based sensitivity indices for models with correlated inputs. Given that Nataf transformation yields several independent parameters sets ([32]), several PCEs can be derived and several sensitivity indices be defined. This is explained in the next subsections.

\subsection{Nataf transformation}

The Nataf transformation ([33, 34, 35]) allows the transformation from original space to mutually independent standard normal one, under Gaussian copula assumption for the joint distribution of the random input vector [36]. 
It does not require the joint probability density function (PDF) of the parameters. However, the marginal PDF of each parameter and the correlation matrix must be known, which is often the case in engineering.

The Nataf transformation is divided into two steps. The first one consists in renormalizing each non normal correlated variable $p_{i}$ into a normal correlated variable $z_{i}$. This renormalization is a CDF matching condition:

$$
z_{i}=\Psi^{-1}\left(H_{i}\left(p_{i}\right)\right), \quad i=1, \ldots, n
$$

where $\Psi^{-1}$ is the inverse CDF of a standard normal variable. Note that $z_{i}$ contains the same information than $p_{i}$.

The correlation matrix $\boldsymbol{\rho}^{\prime}$ of $\mathbf{z}$ is symmetric definite positive and can be decomposed as:

$$
\boldsymbol{\rho}^{\prime}=\boldsymbol{R} \boldsymbol{R}^{T}
$$

where $\boldsymbol{R}$ is the lower triangular matrix obtained from Cholesky decomposition of $\rho^{\prime}$.

The second step of the Nataf transformation consists in transforming the correlated normal variables $\mathbf{z}$ into uncorrelated standard normal variables $\mathbf{u}$ :

$$
\mathbf{u}=\boldsymbol{R}^{-1} \mathbf{z}
$$

It is worthwhile to note that $\boldsymbol{R}^{-1}$ is also lower triangular. As a consequence, $u_{1}$ is proportional to $z_{1}$ and so equivalent to $p_{1}$. The new variable $u_{2}$ is a linear combination of $z_{1}$ and $z_{2}$ but is not correlated to $u_{1}$ (i.e. $p_{1}$ ), so $u_{2}$ represents $p_{2}$ without its correlation with $p_{1}$. In the same manner, $u_{3}$ can be interpreted as the residual part of $p_{3}$ not due to $\left(p_{1}, p_{2}\right)$ and so on... Finally, $u_{n}$ is the proper residual part of $p_{n}$ uncorrelated with the other parameters. 
It can be noted that the computational cost of the Nataf transformation is

related to the dimension of the correlation matrix and is $\frac{1}{3} n^{3}$, where $n$ is the number of parameters.

\subsection{Variance-based sensitivity indices}

Now that we have obtained a set of independent random variables $\mathbf{u}=$ $\left\{u_{1} \ldots, u_{n}\right\}$, their variance-based sensitivity indices can be computed via PCE, as explained in section 3.2. According to the previous discussion, we can infer that:

- $\left(S_{1}, S T_{1}\right)$ the correlation ratio and total effect of $u_{1}$ are those of $p_{1}$. These indices are also called full marginal and total contribution of $p_{1}$ to the variance of $y$ because they include both its correlated and uncorrelated contributions. Indeed, $p_{1}$ is correlated to all the other parameters $\left\{p_{2}, \ldots, p_{n}\right\}$.

- $\left(S_{2}, S T_{2}\right)$ the correlation ratio and total effect of $u_{2}$ are those of $p_{2}$ without its correlative contribution with $p_{1}$. In the sequel, we will use the following notations $\left(S_{2-1}, S T_{2-1}\right)$.

- $\left(S_{n}, S T_{n}\right)$ the correlation ratio and total effect of $u_{n}$ are those of $p_{n}$ without its correlative contribution with the other parameters. They represent $p_{n}$ 's proper contributions and are denoted $\left(S_{n}^{u}, S T_{n}^{u}\right)$.

The sensitivity indices so defined are so interpreted because of their order in the set. Indeed, the above discussion is true for the canonical order, that is, $\mathbf{p}=\left\{p_{1}, p_{2}, \ldots, p_{n}\right\}$. For a different ordering, the new sensitivity indices 
are interpreted in the same way. For instance, with the set $\left\{p_{2}, \ldots, p_{n}, p_{1}\right\}$, $\left(S_{2}, S T_{2}\right)$ and $\left(S_{1}^{u}, S T_{1}^{u}\right)$, among others, can be computed. The complete set $\left(S_{i}, S T_{i}\right)$ and $\left(S_{i}^{u}, S T_{i}^{u}\right)$ can be computed by circularly permuting the parameters order prior to the Nataf transformation.

The sensitivity indices so defined rank within $[0,1]$. If $S T_{i}^{u}=0$ but $S T_{i}$ is high, then it means that $p_{i}$ is only relevant because of its correlations with the other variables. In a sense, this means that the uncertainty of $p_{i}$ can be ignored because its impact on the model response is already embedded in the other inputs uncertainties. On the opposite, if $S T_{i}=0$ but $S T_{i}^{u}$ is high, which occurs in presence of negative correlations, $p_{i}$ is a parameter to account for in the model.

\subsection{Sensitivity analysis of Pacejka model to $(\alpha, B, C, D)$}

\subsubsection{Numerical details}

Let us consider now the set of parameters $(\alpha, B, C, D)$. It is worth noting that the analysis that follows is performed with the same sample generated in section 3.3. Analysis of the parameters sample provides the following correlation matrix:

$$
\hat{\boldsymbol{\rho}}=\left(\begin{array}{cccc}
\alpha & B & C & D \\
1 & 0.00 & 0.00 & 0.00 \\
0.00 & 1 & -0.39 & -0.35 \\
0.00 & -0.39 & 1 & 0.00 \\
0.00 & -0.35 & 0.00 & 1
\end{array}\right) \quad \begin{gathered}
\alpha \\
C \\
D
\end{gathered}
$$

We first start with the parameters set ordering $(\alpha, D, B, C)$. Using the approach described in the previous section, the variance-based sensitivity indices have been computed. The results are summed up in Tables 3 and 4 . 


\begin{tabular}{|c|c|c|c|}
\hline$\alpha$ & $D$ & $B$ & $C$ \\
\hline$\hat{\mathbf{S}}_{\alpha}$ & $\hat{S}_{D-\alpha}$ & $\hat{S}_{B-\alpha D}$ & $\hat{S}_{C}^{u}$ \\
$\mathbf{0 . 4 5}$ & 0.30 & 0.11 & 0.07 \\
\hline$\hat{S}_{\alpha}^{u}$ & $\hat{\mathbf{S}}_{\mathbf{D}}$ & $\hat{S}_{B-D}$ & $\hat{S}_{C-D B}$ \\
0.45 & $\mathbf{0 . 3 0}$ & 0.11 & 0.07 \\
\hline$\hat{S}_{\alpha-B C}$ & $\hat{S}_{D}^{u}$ & $\hat{\mathbf{S}}_{\mathbf{B}}$ & $\hat{S}_{C-B}$ \\
0.46 & 0.44 & $\mathbf{0 . 0 3}$ & 0.03 \\
\hline$\hat{S}_{\alpha-C}$ & $\hat{S}_{D-C \alpha}$ & $\hat{S}_{B}^{u}$ & $\hat{\mathbf{S}}_{\mathbf{C}}$ \\
0.45 & 0.30 & 0.18 & $\mathbf{0 . 0 1}$ \\
\hline
\end{tabular}

Table 3: First-order sensitivity indices

\begin{tabular}{|c|c|c|c|}
\hline$\alpha$ & $D$ & $B$ & $C$ \\
\hline$\hat{\mathbf{S T}}_{\alpha}$ & $\hat{S T}_{D-\alpha}$ & $\hat{S T_{B-\alpha D}}$ & $\hat{S T_{C}^{u}}$ \\
$\mathbf{0 . 5 0}$ & 0.32 & 0.15 & 0.09 \\
\hline$\hat{S T}_{\alpha}^{u}$ & $\hat{\mathbf{S T}}_{\mathbf{D}}$ & $\hat{S T_{B-D}}$ & $\hat{S T_{C}-D B}$ \\
0.50 & $\mathbf{0 . 3 2}$ & 0.15 & 0.09 \\
\hline$\hat{S T}_{\alpha-B C}^{u}$ & $\hat{S T_{D}^{u}}$ & $\hat{\mathbf{S T}}$ & $\hat{S T_{C}}$ \\
0.50 & 0.44 & $\mathbf{0 . 0 7}$ & 0.04 \\
\hline$\hat{S T}_{\alpha-C}$ & $\hat{S T_{D-C \alpha}}$ & $\hat{S T_{B}^{u}}$ & $\hat{\mathbf{S T}}_{\mathbf{C}}$ \\
0.50 & 0.32 & 0.23 & $\mathbf{0 . 0 2}$ \\
\hline
\end{tabular}

Table 4: Total sensitivity indices 


\subsubsection{Results and discussion}

As previously, the results show that $\alpha$ has the highest sensitivity index and therefore is the most relevant parameter. As compared to the previous analysis, its sensitivity indices remain unchanged since it is not correlated to the other parameters. The following most relevant parameter is $D$. Since its contribution is mainly due to its uncorrelated part $\left(\hat{S}_{D}^{u}=0.44\right.$ and $\left.\hat{S}_{D}^{c}=-0.14\right)$ and $D=\mu F_{z}, D$ has almost the same influence as the independent parameter $F_{z}$ on $F_{y}$. As explained previously, $D$, and so $F_{z}$, determines the maximum value of the force $F_{y}$, for a given side slip angle $\alpha$. This explains the relative high contribution of these parameters to the lateral force $F_{y}$. However, there is also a contribution of its correlative part with $B$, which cannot appear in the first study.

The contribution of the uncorrelated part of $B$ is $\hat{S}_{B}^{u}=0.18$ and the one of its correlated part is $\hat{S}_{B}^{c}=-0.15$. It can be worth noting that the correlations being negatives, the sensitivity indices due to the correlation are also negatives. These indices are almost equal, but opposite in sign, leading to a small full marginal index $\left(\hat{S}_{B}=\hat{S}_{B}^{u}+\hat{S}_{B}^{c}=0.03\right)$. This analysis shows the influence of the correlative part of the stiffness factor $B$ to $D$ and consequently to the vertical load $F_{z}$. This cannot be highlighted from the previous analysis of the set of independent parameters $\left(\alpha, F_{z}, K, C\right)$.

It is the same case for the parameter $C\left(\hat{S}_{C}^{u}=0.07, \hat{S}_{C}^{c}=-0.06\right.$ and $\left.\hat{S}_{C}=0.01\right)$. Its influence is low but not negligible, contrary to the previous analysis of independent parameters. This is due to the fact that it is correlated to $B$ that contributes significantly to the output variance. 
As previously, the total indices $\hat{S T}_{i}$ are close to the marginal indices $\hat{S}_{i}$, showing that there are few interactions between the parameters and their influence is mainly due to their individual contribution.

It has been shown that $\alpha$ is the most influential parameter. Lateral force is, in general, a nonlinear function of the slip angle $\alpha$ (figure 2). The relationship between lateral force and slip angle is initially linear with a constant slope determined by the cornering stiffness. In this region, the tread band is supposed to be in complete adhesion with the road surface. As slip angle grows, eventually the force starts to saturate due to the limited friction on the road, entering the nonlinear region and a sliding zone appears. The limit of handling is defined by the maximum available lateral force $\mu F_{z}$. Here, this occurs for $\alpha \approx 15^{\circ}$. The sliding zone increases with the slip angle up to full sliding (see figure 3).

In the following, sensitivity analysis has been performed for the adhesion zone defined by $\alpha \in\left[0 ; 15^{\circ}\right]$ and for the sliding zone defined by $\alpha \in\left[15^{\circ} ; 20^{\circ}\right]$. The other parameters are kept in their initial variation interval. This leads to the sensitivity indices $\hat{S}_{\alpha}=0.5871$ in the adhesion zone and $\hat{S}_{\alpha} \approx 0$ in the sliding zone. It shows that $\alpha$ is more influential in the adhesion zone. In the sliding zone, $F_{y}$ is almost constant with respect to $\alpha$, explaining the insignificant influence of $\alpha$.

In order to plan the experiments for cornering manoeuvre, this study has shown that it is important to sufficiently excite the side slip angle and the vertical load which influences the stiffness factor, especially in the adhesion 


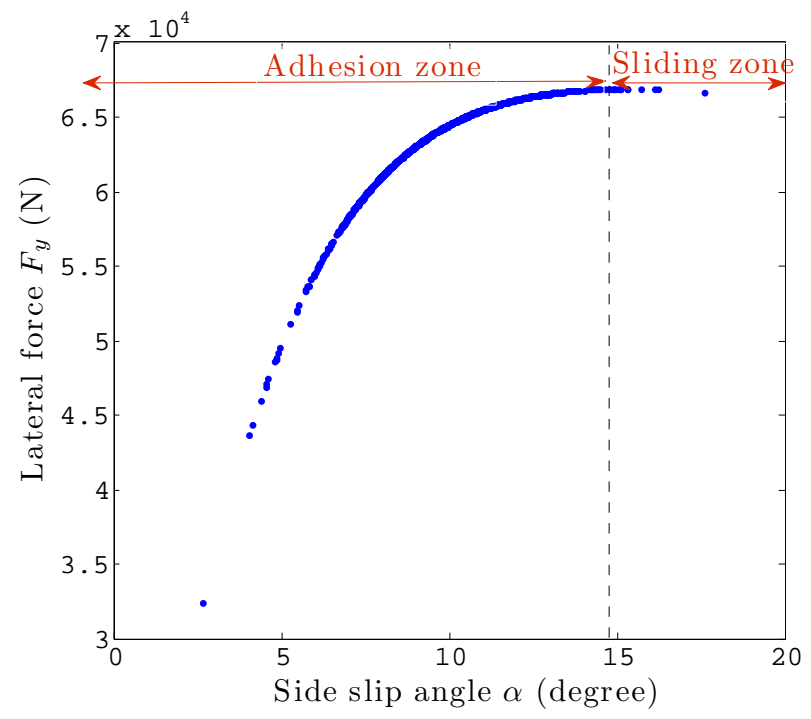

Figure 3: Lateral force vs. side slip angle

zone. Consequently, the following is focused on the adhesion zone.

For the adhesion zone, the $95 \%$ confidence interval of the mean lateral force $\bar{F}_{y}$ is computed and is of $43.5 \%$. Simulations have been made with hypothetic 
reductions of the variation interval of $\alpha$. The results are presented in Table 5.

\begin{tabular}{|c|c|}
\hline Interval reduction of $\alpha$ & $95 \%$ confidence interval of $F_{y}$ \\
\hline $5 \%$ & $32.98 \%$ \\
\hline $10 \%$ & $31.88 \%$ \\
\hline $15 \%$ & $31.5 \%$ \\
\hline
\end{tabular}

Table 5: Hypothetical interval reductions of $\alpha$

It reveals that a reduction of $15 \%$ of the variation interval of $\alpha$ implies a reduction of $12 \%$ of the confidence interval of $\bar{F}_{y}$.

On the other hand, the previous study has shown that the second most influent parameter is $D$, followed by $B$. These parameters are dependent on $F_{z}$ and $K$. Hypothetic reductions of the variation interval of $F_{z}$ have been made combined with the $15 \%$ reduction of $\alpha$. Then, reductions of the interval of $K$ have been made combined with a $15 \%$ reduction of $F_{z}$. The results are shown in Table 6 .

Finally, reducing the variation interval of $C$ does not lead to a significant reduction of $F_{y}$, since $C$ is very few influent on $F_{y}$. In conclusion, acting on the most influent parameters allow to reduce the confidence interval of the mean lateral force of almost the half of its initial value.

The next step will be to extend the sensitivity analysis of the Pacejka model, taking into account the dynamic characteristic of the tire, during transient- 


\begin{tabular}{|c|c|}
\hline Interval reduction of $F_{z}$ & $95 \%$ confidence interval of $F_{y}$ \\
\hline $5 \%$ & $29.39 \%$ \\
\hline $10 \%$ & $28.5 \%$ \\
\hline $15 \%$ & $28 \%$ \\
\hline \hline Interval reduction of $K$ & $95 \%$ confidence interval of $F_{y}$ \\
\hline $5 \%$ & $24.4 \%$ \\
\hline $10 \%$ & $23.6 \%$ \\
\hline $15 \%$ & $22.8 \%$ \\
\hline
\end{tabular}

Table 6: Hypothetical interval reductions of $F_{z}$ and $K$

state, when driving cases as taxiing are considered.

\section{References}

[1] H. B. Pacejka, Tyres and vehicle dynamics, Elsevier, 2006.

[2] R. Rajamani, Vehicle dynamics and control, Springer Verlag, 2005.

[3] M. Gipser, R. Hofer, P. Lugner, Dynamical tyre force response to road unevennesses, Vehicle System Dynamics 27 (1997) 94-108.

[4] M. Blundell, D. Harty, Multibody systems approach to vehicle dynamics, Elsevier, 2005.

[5] E. Bakker, N. Nyborg, H. Pacejka, Tyre modelling for use in vehicle dynamics studies, 1987. 
[6] R. I. Cukier, R. I. Levine, K. E. Shuler, Nonlinear sensitivity analysis of multiparameter model systems, Journal of Computational Physics 26 (1978) 1-42.

[7] I. M. Sobol, Sensitivity estimates for nonlinear mathematical models, Mathematical Modelling and Computing Experiments 1 (1993) 407-414.

[8] A. Saltelli, S. Tarantola, K. Chan, A quantitative model independent method for global sensitivity analysis of model output, Technometrics 41 (1999) 39-56.

[9] H. Frey, S. R. Patil, Identification and review of sensitivity analysis methods, Risk Analysis 22 (3) (2002) 553-578.

[10] A. Saltelli, Sensitivity analysis for importance assessment, Risk Analysis 22 (3) (2002) 579-590.

[11] A. Saltelli, S. Tarantola, F. Campolongo, N. Ratto, Sensitivity Analysis in Practice. A Guide to Assessing Scientific Models, Wiley, 2004.

[12] G. Blatman, B. Sudret, Sparse polynomial chaos expansion and adaptive stochastic finite element using a regression approach, Comptes rendus de Mécanique 336 (6) (2008) 518-523.

[13] J. Jacques, C. Lavergne, N. Devictor, Sensitivity analysis in presence of model uncertainty and correlated inputs, Reliability Engineering \& System Safety 91 (2006) 1126-1134.

[14] T. A. Mara, S. Tarantola, Variance-based sensitivity indices for models 
with dependent inputs, Reliability Engineering and System Safety 107 (2012) 115-121.

[15] N. Wiener, The homogeneous chaos, American Journal of Mathematics 60 (4) (1938) 897-936.

[16] A. Nataf, Détermination des distributions dont les marges sont données, Comptes Rendus de l'Académie des Sciences 225 (1962) 42-43.

[17] M. Rosenblatt, Remarks on the multivariate transformation, Annals of Mathematics and Statistic 43 (1952) 470-472.

[18] B. Sudret, Global sensitivity analysis using polynomial chaos expansion, Reliability Engineering \& System Safety 93 (2008) 964-979.

[19] S. Rahman, Global sensitivity analysis by polynomial dimensional decomposition, Reliability Engineering and System Safety 86 (7) (2011) $825-837$.

[20] R. H. Cameron, W. T. Martin, The orthogonal development of nonlinear functionals in series of Fourier-Hermite functionals, Annals of Mathematics (1947) 48:385.

[21] D. Xiu, G. Karniadakis, The Wiener-Askey polynomials chaos for stochastic differential equations, Journal of Scientific Computing 24 (2) (2002) 619-644.

[22] G. Blatman, B. Sudret, Efficient computation of global sensitivity indices using sparse polynomial chaos expansions, Reliability Engineering and System Safety 95 (2010) 1216-1229. 
[23] M. Berveiller, B. Sudret, M. Lemaire, Stochastic finite element: a non intrusive approach by regression, European Journal of Computational Mechanics 15 (1-3) (2006) 81-92.

[24] B. Sudret, M. Berveiller, M. Lemaire, Application of a stochastic finite element procedure to reliability analysis, European Journal of Computational Mechanics 15 (7-8) (2006) 825-866.

[25] T. Crestaux, O. Le Maitre, J. Martinez, Polynomial chaos expansion for sensitivity analysis, Reliability Engineering \& System Safety 94 (2009) $1161-1172$.

[26] M. Eldred, Recent advances in non-intrusive polynomial chaos and stochastic collocation methods for uncertainty analysis and design, Tech. rep., 50th AIAA/ASME/ASCE/AHS/ASC Structures, Structural Dynamics, and Materials Conference (2009).

[27] M. D. McKay, J. D. Morrison, S. C. Upton, Evaluating prediction uncertainty in simulation models, Computer Physics Communications 117 (12) (1999) 239-245.

[28] M. S. Constantine, P. Eldred, E. Phipps, Sparse pseudo spectral approximation method, Computer Methods in Applied Mechanics and Engineering 229-232 (2012) 1-12.

[29] H. Niederreiter, Random number generation and quasi-Monte-Carlo methods, in: SIAM CBMS-NSF Regional Conference Series in Applied Mathematics, Philadelphia., 1992. 
[30] J. Helton, J. Johnson, C. Sallaberry, C. Storlie, Survey of samplingbased methods for uncertainty and sensitivity analysis, Reliability Engineering \& System Safety 91 (2006) 1175-1209.

[31] R. I. Iman, W. J. Conover, A distribution-free approach to inducing rank correlation among input variables, Communications in Statistics: Simulation and Computation 11 (1982) 311-334.

[32] R. Lebrun, A. Dutfoy, Do Rosenblatt and Nataf isoprobabilistic transformations really differ?, Probabilistic Engineering Mechanics 24 (2009) $577-584$.

[33] P. L. Liu, A. D. Kiureghian, A multivariate distribution models with prescribed marginals and covariances, Probabilistic Engineering Mechanics 112 (1986) 85-104.

[34] H. S. Li, Z. Z. Lü, X. K. Yuan, Nataf transformation based point estimate method, Chinese Science Bulletin 53 (17) (2008) 2586-2592.

[35] Y. Noh, K. K. Choi, L. Du, Reliability-based design optimization of problems with correlated input variables using a Gaussian Copula, Structural and Multidisciplinary Optimization 38 (2009) 1-16.

[36] R. Lebrun, A. Dutfoy, An innovating analysis of the nataf transformation from the copula viewpoint, Probabilistic Engineering Mechanics 24 (3) (2009) 312-320. 